\title{
Monster anatomy
}

\section{Jin-Beom Bae, Kimyeong Lee and Sungjay Lee}

Korea Institute for Advanced Study, 85 Hoegiro, Dongdaemun-Gu, Seoul 02455, Korea

E-mail: jinbeom@kias.re.kr, klee@kias.re.kr, sjlee@kias.re.kr

ABStract: We investigate the two-dimensional conformal field theories (CFTs) of $c=\frac{47}{2}$, $c=\frac{116}{5}$ and $c=23$ 'dual' to the critical Ising model, the three state Potts model and the tensor product of two Ising models, respectively. We argue that these CFTs exhibit moonshines for the double covering of the baby Monster group, $2 \cdot \mathbb{B}$, the triple covering of the largest Fischer group, $3 \cdot \mathrm{Fi}_{24}^{\prime}$ and multiple-covering of the second largest Conway group, $2 \cdot 2^{1+22} \cdot \mathrm{Co}_{2}$. Various twined characters are shown to satisfy generalized bilinear relations involving Mckay-Thompson series. We also rediscover that the 'self-dual' twodimensional bosonic conformal field theory of $c=12$ has the Conway group $\mathrm{Co}_{0} \simeq 2 \cdot \mathrm{Co}_{1}$ as an automorphism group.

Keywords: Conformal and W Symmetry, Field Theories in Lower Dimensions

ArXiv EPrint: 1811.12263 


\section{Contents}

1 Introduction 1

2 Dual of the Ising model and the Baby Monster 3

3 Dual of the three-state Potts model and $3 \cdot \mathrm{Fi}_{24}^{\prime} \quad 6$

4 Dual of the critical Ising ${ }^{2}$ and $2 \cdot 2^{1+22} \cdot \mathrm{Co}_{2}$

5 Self-dual RCFT and $2 \cdot \mathrm{Co}_{1} \quad 11$

6 Discussion 13

$\begin{array}{ll}\text { A Dimension of the irreducible representations } & 15\end{array}$

B Character tables of $2 \cdot \mathbb{B}$ and $3 \cdot \mathrm{Fi}_{24}^{\prime} \quad 16$

$\begin{array}{ll}\text { C Generalized bilinear relations } & 18\end{array}$

$\begin{array}{lll}\text { C.1 } 2 \cdot \mathbb{B} & 18\end{array}$

$\begin{array}{lll}\text { C.2 } & 3 \cdot \mathrm{Fi}_{24}^{\prime} & 18\end{array}$

\section{Introduction}

Mckay and Thompson's remarkable observation between the monster group $\mathbb{M}$ and the modular objects, especially, the $j$-invariant, motivated the study of the so-called 'Monstrous Moonshine' in [1]. Each Fourier coefficient of the modular invariant $j(\tau)-744\left(q=e^{2 \pi i \tau}\right)$, which can describe the partition function of $c=24$ chiral CFT,

$$
j(\tau)-744=\frac{1}{q}+196884 q+21493760 q^{2}+\cdots,
$$

can be decomposed into the dimension of the irreducible representation of the monster group M. Frenkel, Lepowsky and Meurman [2] provided a (heuristic) derviation of the Monstrous moonshine from an explicit construction of the chiral CFT based on the Leech lattice followed by a $\mathbb{Z}_{2}$ orbifold. Many examples of the generalizations of moonshine phenomena with different sporadic groups have been uncovered in the last decades [3-11].

In this article, we utilize a holomorphic bilinear relation [12] to further explore a new class of moonshine phenomena. It has been observed recently that characters $f_{i}(\tau)$ of a certain rational CFT with central charge $c$ obey an intriguing bilinear relation giving a modular invariant $j(\tau)$,

$$
\sum_{i=0}^{n-1} f_{i}(\tau) \tilde{f}_{i}(\tau)=j(\tau)-744
$$


where $\tilde{f}_{i}(\tau)$ can be interpreted as characters of a 'dual' rational CFT with central charge $(24-c)$. For instance, the critical Ising model with $c=\frac{1}{2}$ and a rational CFT with $c=\frac{47}{2}$ satisfies the bilinear relation. Another example is a pair of rational CFTs of $c=8$ and $c=16$ having no Kac-Moody symmetry but finite group symmetry [13]. Further examples can be found in [14].

The rational CFT with $c=\frac{47}{2}$ dual to the critical Ising model exhibits Moonshine for the baby Monster group, second largest sporadic group. It is challenging to search for a dual rational CFT showing Moonshine for the sporadic groups other than the baby Monster group. The search first requires an explicit $q$-expansion of each character in two rational CFTs of dual pair. To do so, we make use of a modular-invariant differential equation (MDE) of the form below [15]

$$
\left[\mathcal{D}_{\tau}^{n}+\sum_{k=0}^{n-1} \phi_{2(n-k)}(\tau) \mathcal{D}_{\tau}^{k}\right] f(\tau)=0,
$$

where $\mathcal{D}_{\tau}$ denotes the Serre derivative acting on a modular form of weight $r$,

$$
\mathcal{D}_{\tau} \equiv \partial_{\tau}-\frac{1}{6} i \pi r E_{2}(\tau),
$$

and $\phi_{k}(\tau)$ are modular forms of weight $k$. The MDE can be used to explore the space of rational CFTs. This is because solutions to an MDE, which furnish a finite-dimensional representation of $\operatorname{SL}(2, \mathbb{Z})$, can play a role as candidate characters $f_{i}(\tau)(i=0,1,2, \ldots, n-1)$ in a rational CFT. One can show from (1.3) that the conformal weights $h_{i}$ of primaries and the central charge $c$ of a candidate RCFT have to satisfy the relation below

$$
\sum_{i=0}^{n-1}\left[h_{i}-\frac{c}{24}\right]=\frac{n(n-1)}{12}-\frac{l}{6},
$$

where $l$ is a non-negative integer other than 1. (1.5) implies that a rational CFT can be characterized by conformal weights $h_{i}$ and a number $n$ of primaries, the central charge $c$ and an integer $l$. When $\left(\left\{h_{i}\right\}, n, c, l\right)$ of a rational CFT are related to $\left(\left\{\tilde{h}_{i}\right\}, n, \tilde{c}, \tilde{l}\right)$ of another rational CFT as follows

$$
h_{0}=\tilde{h}_{0}=0, \quad h_{i}+\tilde{h}_{i}=2 \text { for } i \neq 0,
$$

and

$$
c+\tilde{c}=24, \quad l+\tilde{l}=(n-3)(n-4),
$$

the characters of two rational CFTs can obey the bilinear relation (1.2). Namely, one rational CFT is dual to the other.

We analyze rational CFTs dual to the three-state Potts model and to the product of two Ising models. Interestingly enough, our results can propose that the dual rational CFTs show novel connections to the triple covering of the largest Fischer group, $3 \cdot \mathrm{Fi}_{24}^{\prime}$ and the multiple-covering of the second largest Conway group, $2 \cdot 2^{1+22} \cdot \mathrm{Co}_{2}$, respectively. Note 


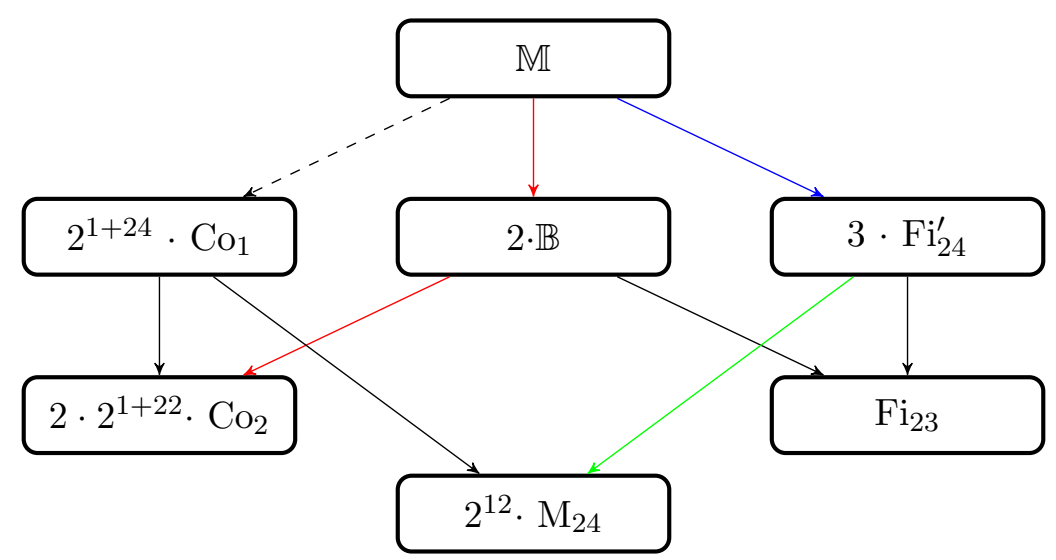

Figure 1. Partial flows of maximal subgroups from the monster group $\mathbb{M}$ [16]. Each arrow from $A$ to $\mathrm{B}$ implies that $\mathrm{B}$ is a maximal subgroup of $\mathrm{A}$.

that $3 \cdot \mathrm{Fi}_{24}^{\prime}$ is a maximal subgroup of $\mathbb{M}$ described in figure 1 . We also observe that the self-dual theory of $c=12$ has the largest Conway group $\mathrm{Co}_{0}$ as an automorphism group. In fact, we can show that the self-dual theory can be identified as the GSO projection of the well-known $\mathcal{N}=1$ superconformal extremal CFT of $c=12[2,17]$. Our observations suggest that the subgroup decomposition along the red and the blue arrows in figure 1 can be realized by the holomorphic bilinear relation. The moonshines for $2 \cdot \mathbb{B}$ in baby Monster CFT and $3 \cdot \mathrm{Fi}_{24}^{\prime}$ in $c=\frac{116}{5} \mathrm{CFT}$ are further supported by generalized bilinear relations involving Mckay-Thompson series. The details will be shown in section 2 and section 3 .

\section{Dual of the Ising model and the Baby Monster}

The simplest unitary minimal model $\mathcal{M}(4,3)$ describes the critical point of the secondorder phase transition of the Ising model. The critical Ising model has the identity $\mathbf{1}$, the energy density $\epsilon$ and the spin $\sigma$ whose scaling dimensions are $\Delta=0,1, \frac{1}{8}$ respectively. Assuming three operators have no spin, one can relate them with allowed primary fields $\phi_{1,1}, \phi_{2,1}, \phi_{2,2}$ of weight $h=0, \frac{1}{2}, \frac{1}{16}$ in $\mathcal{M}(4,3)$ as follows

$$
\begin{aligned}
1 & \leftrightarrow \phi_{1,1} \\
\epsilon & \leftrightarrow \phi_{2,1} \\
\sigma & \leftrightarrow \phi_{2,2}
\end{aligned}
$$

The $c=\frac{1}{2}$ Virasoro characters for three primary fields are

$$
\begin{aligned}
& f_{0}(\tau)=\frac{1}{2}\left(\sqrt{\frac{\vartheta_{3}(\tau)}{\eta(\tau)}}+\sqrt{\frac{\vartheta_{4}(\tau)}{\eta(\tau)}}\right), \\
& f_{\epsilon}(\tau)=\frac{1}{2}\left(\sqrt{\frac{\vartheta_{3}(\tau)}{\eta(\tau)}}-\sqrt{\frac{\vartheta_{4}(\tau)}{\eta(\tau)}}\right), \\
& f_{\sigma}(\tau)=\frac{1}{\sqrt{2}} \sqrt{\frac{\vartheta_{2}(\tau)}{\eta(\tau)}} .
\end{aligned}
$$


These characters transform into one another under $\mathrm{SL}(2, \mathbb{Z})$. In particular, the modular S-matrix is

$$
\left(\begin{array}{l}
f_{0}(-1 / \tau) \\
f_{\epsilon}(-1 / \tau) \\
f_{\sigma}(-1 / \tau)
\end{array}\right)=\frac{1}{2}\left(\begin{array}{ccc}
1 & 1 & \sqrt{2} \\
1 & 1 & -\sqrt{2} \\
\sqrt{2} & -\sqrt{2} & 0
\end{array}\right)\left(\begin{array}{l}
f_{0}(\tau) \\
f_{\epsilon}(\tau) \\
f_{\sigma}(\tau)
\end{array}\right)
$$

It has been shown recently in [12] that the characters of the critical Ising model obey an intriguing bilinear relation,

$$
j(\tau)-744=f_{0}(\tau) \cdot \tilde{f}_{0}(\tau)+f_{\epsilon}(\tau) \cdot \tilde{f}_{\epsilon}(\tau)+f_{\sigma}(\tau) \cdot \tilde{f}_{\sigma}(\tau),
$$

where $j(\tau)-744$ is the Monster module,

$$
j(\tau)=\frac{12^{3} E_{4}^{3}(\tau)}{E_{4}^{3}(\tau)-E_{6}^{2}(\tau)}=\frac{1}{q}+744+196884 q+21493760 q^{2}+\cdots .
$$

Here $\tilde{f}_{0}, \tilde{f}_{\sigma}$ and $\tilde{f}_{\epsilon}$ are three independent solutions to a third order modular differential equation

$$
0=\left[\mathcal{D}_{\tau}^{3}+\frac{2315 \pi^{2}}{576} E_{4}(\tau) \mathcal{D}_{\tau}-i \frac{27025 \pi^{3}}{6912} E_{6}(\tau)\right] \tilde{f}
$$

and can be expanded in powers of $q$ as follow.

$$
\begin{aligned}
& \tilde{f}_{0}(\tau)=q^{-\frac{47}{48}}\left(1+96256^{\prime} q^{2}+9646891 q^{3}+366845011 q^{4}+\cdots\right) \\
& \tilde{f}_{\epsilon}(\tau)=q^{\frac{25}{48}}\left(4371+1143745 q+64680601 q^{2}+1829005611 q^{3}+\cdots\right) \\
& \tilde{f}_{\sigma}(\tau)=q^{\frac{23}{24}}\left(96256+10602496 q+420831232 q^{2}+9685952512 q^{3}+\cdots\right) .
\end{aligned}
$$

In fact, (2.7) can be identified as three characters of conformal weights $h=0, \frac{31}{16}, \frac{3}{2}$ in the dual RCFT with $c=\frac{47}{2}$.

Since the $j(\tau)$ is invariant under $\operatorname{SL}(2, \mathbb{Z})$, the modular S-matrix of the dual RCFT with $c=\frac{47}{2}$, often referred to as the baby Monster CFT, obtained from that of the critical Ising model, namely

$$
\left(\begin{array}{c}
\tilde{f}_{0}(-1 / \tau) \\
\tilde{f}_{\epsilon}(-1 / \tau) \\
\tilde{f}_{\sigma}(-1 / \tau)
\end{array}\right)=\frac{1}{2}\left(\begin{array}{ccc}
1 & 1 & \sqrt{2} \\
1 & 1 & -\sqrt{2} \\
\sqrt{2} & -\sqrt{2} & 0
\end{array}\right)\left(\begin{array}{c}
\tilde{f}_{0}(\tau) \\
\tilde{f}_{\epsilon}(\tau) \\
\tilde{f}_{\sigma}(\tau)
\end{array}\right) .
$$

In addition, the Verlinde formulae implies that these two RCFTs also share the same fusion rule algebra. From (2.8), the modular invariant torus partition function of the baby Monster CFT has to be

$$
Z_{c=\frac{47}{2}}(\tau, \bar{\tau})=\tilde{f}_{0}(\tau) \overline{\tilde{f}}_{0}(\bar{\tau})+\tilde{f}_{\epsilon}(\tau) \overline{\tilde{f}}_{\epsilon}(\bar{\tau})+\tilde{f}_{\sigma}(\tau) \overline{\tilde{f}}_{\sigma}(\bar{\tau})
$$

which agrees with numerical results in [13].

It is known that dual RCFT with $c=\frac{47}{2}$ has baby Monster group $\mathbb{B}$ as a finite group symmetry [12], because (2.7) are identical to the characters of modules $V \mathbb{B}_{(0)}^{\natural}, V \mathbb{B}_{(1)}^{\natural}$ and 
$V \mathbb{B}_{(2)}^{\natural}$ in [18], respectively. In this paper, we conjecture that the finite group symmetry of $c=\frac{47}{2}$ RCFT can be promoted to the double covering of baby Monster group $2 \cdot \mathbb{B}$. As a demonstration, one can show that each coefficient in (2.7) can be expressed as a sum of dimensions of irreducible representations of $2 \cdot \mathbb{B}$, e.g.

$$
\begin{aligned}
4371 & =\mathbf{4 3 7 1}, \quad 96256^{\prime}=\mathbf{1} \oplus \mathbf{9 6 2 5 5}, \quad 96256=\mathbf{9 6 2 5 6}, \\
9646891 & =\mathbf{1} \oplus \mathbf{9 6 2 5 5} \oplus \mathbf{9 5 5 0 6 3 5}, \quad 1143745=\mathbf{4 3 7 1} \oplus \mathbf{1 1 3 9 3 7 4}, \\
10602496 & =\mathbf{9 6 2 5 6} \oplus \mathbf{1 0 5 0 6 2 4 0}, \\
366845011 & =2 \cdot \mathbf{1} \oplus 2 \cdot \mathbf{9 6 2 5 5} \oplus \mathbf{9 4 5 8 7 5 0} \oplus \mathbf{9 5 5 0 6 3 5} \oplus \mathbf{3 4 7 6 4 3 1 1 4}, \\
64680601 & =2 \cdot \mathbf{4 3 7 1} \oplus \mathbf{1 1 3 9 3 7 4} \oplus \mathbf{6 3 5 3 2 4 8 5}, \\
420831232 & =2 \cdot \mathbf{9 6 2 5 6} \oplus \mathbf{1 0 5 0 6 2 4 0} \oplus \mathbf{4 1 0 1 3 2 4 8 0} .
\end{aligned}
$$

Note that $\mathbf{1}$ in the second line of (2.10) can be identified as the Virasoro descendent of the vacuum.

We apply a refined test proposed in $[19,20]$ to provide further supporting evidence that $c=\frac{47}{2} \mathrm{CFT}$ exhibit moonshine for $2 \cdot \mathbb{B}$. To this end, we introduce the twined character,

$$
\tilde{f}_{i}^{g}(\tau)=\operatorname{Tr}_{\mathcal{H}_{i}}\left[g \cdot q^{h-\frac{c}{24}}\right] .
$$

where $g$ is a group element of $2 \cdot \mathbb{B}$ and the trace is taken over all states in the Hilbert space $\mathcal{H}_{i}$, that consist of primary state of weight $h_{i}$ and its descendants. As illustrated in [19], it is straightforward to obtain the twined characters using the character table of $2 \cdot \mathbb{B}$ in appendix B. For instance, let us consider the twined characters for $g=2 C$. Explicitly, the first term in $\tilde{f}_{\sigma}(\tau), \mathbf{9 6 2 5 6}$, is replaced by 2048. In this way, one can check that the twined characters are given by

$$
\begin{aligned}
& \tilde{f}_{0}^{2 C}(q)=q^{-\frac{47}{48}}\left(1+2048 q^{2}+37675 q^{3}+470099 q^{4}+\cdots\right), \\
& \tilde{f}_{\epsilon}^{2 C}(q)=q^{\frac{25}{48}}\left(275+9153 q+144025 q^{2}+\cdots\right) \\
& \tilde{f}_{\sigma}^{2 C}(q)=q^{\frac{23}{24}}\left(2048+47104 q+565248 q^{2}+\cdots\right) .
\end{aligned}
$$

Intriguingly, we find that (2.12) satisfy a bilinear relation of the form

$$
j^{2 A}(\tau)=f_{0}(\tau) \cdot \tilde{f}_{0}^{2 C}(\tau)+f_{\epsilon}(\tau) \cdot \tilde{f}_{\epsilon}^{2 C}(\tau)+f_{\sigma}(\tau) \cdot \tilde{f}_{\sigma}^{2 C}(\tau),
$$

where $j^{2 A}(\tau)$ is Mckay-Thompson series of class $2 \mathrm{~A}$,

$$
\begin{aligned}
j^{2 A}(\tau) & =\frac{\eta(q)^{24}}{\eta\left(q^{2}\right)^{24}}+\frac{2^{12} \eta\left(q^{2}\right)^{24}}{\eta(q)^{24}}+24 \\
& =\frac{1}{q}+4372 q+96256 q^{2}+1240002 q^{3}+10698752 q^{4}+\cdots .
\end{aligned}
$$

In appendix $\mathrm{C}$, we present list of the generalized bilinear relations for various $g \in 2 \cdot \mathbb{B}$. Combined with the characters of the Ising model, all the twined characters we investigated constitutes Mckay-Thompson series of certain class. Sometimes, combination of the twined characters for different group elements and characters of the critical Ising model 
yields Mckay-Thompson series of identical class. More precisely, even if we replace 2C characters to $2 \mathrm{~A}$ (or $2 \mathrm{~B}$ ) characters in the right-hand side of (2.13), the left-hand side of (2.13) still remained as $j^{2 A}(\tau)$. Note that the decompositions in (2.10) are consistent to bilinear relations.

\section{Dual of the three-state Potts model and $3 \cdot \mathrm{Fi}_{24}^{\prime}$}

We now make use of the bilinear relations but with different pairs of characters to look for new RCFTs related to the other sporadic groups. In particular, we propose in this section that a dual CFT of the three-state Potts model has the triple covering of the largest Fischer group $\mathrm{Fi}_{24}^{\prime}$ as an automorphism group.

It is known that the three-state Potts model at the critical point can be described as a "subset" of the minimal model $\mathcal{M}(6,5)$ with $c=\frac{4}{5}$ containing ten primary fields $\phi_{(r, s)}$ of conformal weights

$$
h_{r, s}=\frac{(6 r-5 s)^{2}-1}{120}
$$

where $1 \leq r<5,1 \leq s<6$ and $(r, s) \simeq(5-r, 6-s)$. The $c=\frac{4}{5}$ Virasoro characters labelled by two integers $(r, s)$ are given by

$$
f_{r, s}^{(5)}(q)=\frac{1}{\eta(q)} \sum_{n \in \mathbb{Z}}\left[q^{\frac{(60 n+6 r-5 s)^{2}}{120}}-q^{\frac{(60 n+6 r+5 s)^{2}}{120}}\right] .
$$

Some but not all of these primary fields are present in the critical three-state Potts model. One can indeed show that a subset of the ten primary fields are closes under the fusion rules, which leads to a non-diagonal modular invariant partition function

$$
Z=\sum_{r=1,2}\left|f_{r, 1}^{(5)}+f_{r, 5}^{(5)}\right|^{2}+2\left|f_{r, 3}^{(5)}\right|^{2}
$$

(3.3) is the partition function of the three-state Potts model, which implies that only $\phi_{r, s}$ and two copies of $\phi_{r, 3}$ with $r=1,2$ and $s=1,5$ are present in the theory. Notice also that the three-state Potts model has $\mathbb{Z}_{3}$ symmetry under which two copies of $\phi_{r, 3}$ for each $r$ transform differently. This $\mathbb{Z}_{3}$ symmetry plays a key role to have well-defined fusion rules of the three-states Potts model [21].

It is natural from (3.3) to define the characters of the critical three-state Potts model as follows,

$$
\begin{aligned}
& f_{0}(\tau)=f_{1,1}^{(5)}(\tau)+f_{1,5}^{(5)}(\tau), \\
& f_{1}(\tau)=f_{2,1}^{(5)}(\tau)+f_{2,5}^{(5)}(\tau), \\
& f_{2}(\tau)=f_{2}^{\prime}(\tau)=f_{1,3}^{(5)}(\tau), \\
& f_{3}(\tau)=f_{3}^{\prime}(\tau)=f_{2,3}^{(5)}(\tau) .
\end{aligned}
$$


The modular S-matrix of the model then becomes

$$
\left(\begin{array}{l}
f_{0}(-1 / \tau) \\
f_{1}(-1 / \tau) \\
f_{2}(-1 / \tau) \\
f_{2}^{\prime}(-1 / \tau) \\
f_{3}(-1 / \tau) \\
f_{3}^{\prime}(-1 / \tau)
\end{array}\right)=\frac{2}{\sqrt{15}}\left(\begin{array}{cccccc}
-s_{1} & s_{2} & -s_{1} & -s_{1} & s_{2} & s_{2} \\
s_{2} & s_{1} & s_{2} & s_{2} & s_{1} & s_{1} \\
-s_{1} & s_{2} & -\omega s_{1} & -\omega^{2} s_{1} & \omega s_{2} & \omega^{2} s_{2} \\
-s_{1} & s_{2} & -\omega^{2} s_{1} & -\omega s_{1} & \omega^{2} s_{2} & \omega s_{2} \\
s_{2} & s_{1} & \omega s_{2} & \omega^{2} s_{2} & \omega s_{1} & \omega^{2} s_{1} \\
s_{2} & s_{1} & \omega^{2} s_{2} & \omega s_{2} & \omega^{2} s_{1} & \omega s_{1}
\end{array}\right)\left(\begin{array}{l}
f_{0}(\tau) \\
f_{1}(\tau) \\
f_{2}(\tau) \\
f_{2}^{\prime}(\tau) \\
f_{3}(\tau) \\
f_{3}^{\prime}(\tau)
\end{array}\right) .
$$

where $s_{1}=\sin \left(\frac{6 \pi}{5}\right), s_{2}=\sin \left(\frac{12 \pi}{5}\right)$ and $\omega=e^{\frac{2 \pi i}{3}}$.

One can show that the characters (3.4) satisfy a bilinear relation

$$
\begin{aligned}
j(\tau)-744= & f_{0}(\tau) \tilde{f}_{0}(\tau)+f_{1}(\tau) \tilde{f}_{1}(\tau)+f_{2}(\tau) \tilde{f}_{2}(\tau)+f_{2}^{\prime}(\tau) \tilde{f}_{2}^{\prime}(\tau) \\
& +f_{3}(\tau) \tilde{f}_{3}(\tau)+f_{3}^{\prime}(\tau) \tilde{f}_{3}^{\prime}(\tau),
\end{aligned}
$$

where the four characters of a dual theory $\tilde{f}_{i}(\tau)$ are the solutions to a fourth order differential equation,

$$
\left[\mathcal{D}_{\tau}^{4}+\mu_{1} E_{4}(\tau) \mathcal{D}_{\tau}^{2}+\mu_{2} E_{6}(\tau) \mathcal{D}_{\tau}+\mu_{3} E_{4}^{2}(\tau)\right] \tilde{f}_{i}(\tau)=0
$$

Here, we denote by $\tilde{f}_{1}(\tau), \tilde{f}_{2}(\tau), \tilde{f}_{3}(\tau)$ and $\tilde{f}_{4}(\tau)$ characters of weights $h=0, \frac{8}{5}, \frac{4}{3}$ and $\frac{29}{15}$, respectively. We further use the $q$-expansion of $\tilde{f}_{i}(\tau)$

$$
\tilde{f}_{i}(\tau)=q^{\left(h_{i}-\frac{c}{24}\right)}\left(a_{0}+a_{1} q+a_{2} q^{2}+a_{3} q^{3}+\cdots\right),
$$

to fix free parameters $\mu_{i}$ in (3.7). The fourth order differential equation of our interest then becomes

$$
0=\left[\mathcal{D}_{\tau}^{4}+\frac{907 \pi^{2}}{225} E_{4}(\tau) \mathcal{D}_{\tau}^{2}-i \frac{4289 \pi^{3}}{675} E_{6}(\tau) \mathcal{D}_{\tau}-\frac{175769 \pi^{4}}{50625} E_{4}^{2}(\tau)\right] \tilde{f}(\tau) .
$$

Now, It is easy to see that the solutions of (3.9) have the $q$-expansion

$$
\begin{aligned}
& \tilde{f}_{0}(\tau)=q^{-\frac{29}{30}}\left(1+57478 q^{2}+5477520 q^{3}+201424111 q^{4}+\cdots\right), \\
& \tilde{f}_{1}(\tau)=q^{\frac{19}{30}}\left(8671+1675504 q+83293626 q^{2}+2175548448 q^{3}+\cdots\right), \\
& \tilde{f}_{2}(\tau)=\tilde{f}_{2}^{\prime}(\tau)=q^{\frac{11}{30}}\left(783+306936 q+19648602 q^{2}+\cdots\right) \\
& \tilde{f}_{3}(\tau)=\tilde{f}_{3}^{\prime}(\tau)=q^{\frac{29}{30}}\left(64584+6789393 q+261202536 q^{2}+\cdots\right) .
\end{aligned}
$$

From the bilinear relation (3.6), one can read that the modular S-matrix of the dual theory is

$$
\left(\begin{array}{c}
\tilde{f}_{0}(-1 / \tau) \\
\tilde{f}_{1}(-1 / \tau) \\
\tilde{f}_{2}(-1 / \tau) \\
\tilde{f}_{2}^{\prime}(-1 / \tau) \\
\tilde{f}_{3}(-1 / \tau) \\
\tilde{f}_{3}^{\prime}(-1 / \tau)
\end{array}\right)=\frac{2}{\sqrt{15}}\left(\begin{array}{cccccc}
-s_{1} & s_{2} & -s_{1} & -s_{1} & s_{2} & s_{2} \\
s_{2} & s_{1} & s_{2} & s_{2} & s_{1} & s_{1} \\
-s_{1} & s_{2} & -\omega^{2} s_{1} & -\omega s_{1} & \omega^{2} s_{2} & \omega s_{2} \\
-s_{1} & s_{2} & -\omega s_{1} & -\omega^{2} s_{1} & \omega s_{2} & \omega^{2} s_{2} \\
s_{2} & s_{1} & \omega^{2} s_{2} & \omega s_{2} & \omega^{2} s_{1} & \omega s_{1} \\
s_{2} & s_{1} & \omega s_{2} & \omega^{2} s_{2} & \omega s_{1} & \omega^{2} s_{1}
\end{array}\right)\left(\begin{array}{c}
\tilde{f}_{0}(\tau) \\
\tilde{f}_{1}(\tau) \\
\tilde{f}_{2}(\tau) \\
\tilde{f}_{2}^{\prime}(\tau) \\
\tilde{f}_{3}(\tau) \\
\tilde{f}_{3}^{\prime}(\tau)
\end{array}\right) .
$$


We can also verify that the dual theory with $3 \cdot \mathrm{Fi}_{24}^{\prime}$ symmetry has positive integer fusion coefficients, obtained from the above S-matrix via the Verlinde formula.

Notice that each coefficient of (3.10) can be expressed as a sum of dimensions of representations of $3 \cdot \mathrm{Fi}_{24}^{\prime}$, the triple covering of the largest Fischer group. $3 \cdot \mathrm{Fi}_{24}^{\prime}$ is one of the maximal subgroup of the Monster group, and is of order $2^{21} \cdot 3^{17} \cdot 5^{2} \cdot 7^{3} \cdot 11 \cdot 13 \cdot 17 \cdot 23 \cdot 29$. It has 256 irreducible representations including $\mathbf{7 8 3 , 8 6 7 1}$ and $\mathbf{6 4 5 8 4}$ that agree with the first coefficient of $\tilde{f}_{2}(\tau), \tilde{f}_{1}(\tau)$ and $\tilde{f}_{3}(\tau)$. One can also show that

$$
\begin{aligned}
57478 & =\mathbf{1} \oplus \mathbf{5 7 4 7 7}, \quad 1675504=\mathbf{8 6 7 1} \oplus \mathbf{1 6 6 6 8 3 3}, \\
306936 & =\mathbf{7 8 3} \oplus \mathbf{3 0 6 1 5 3}, \quad 6789393=\mathbf{6 4 5 8 4} \oplus \mathbf{6 7 2 4 8 0 9}, \\
5477520 & =\mathbf{1} \oplus \mathbf{5 7 4 7 7} \oplus \mathbf{5 5 5 6 1 1} \oplus \mathbf{4 8 6 4 4 3 1}
\end{aligned}
$$

where the first term of each line in (3.12) can be understood as the Virasoro descendant of the corresponding primary field.

Now we will find the twined characters of $c=\frac{116}{5}$ CFT and examine if they form a bilinear relation analogous to (2.13). For instance, the twined characters for $g=2 A$ are given by

$$
\begin{aligned}
& \tilde{f}_{0}^{2 A}(q)=q^{-\frac{29}{30}}\left(1+1158 q^{2}+20112 q^{3}+\cdots\right), \\
& \tilde{f}_{1}^{2 A}(q)=q^{\frac{19}{30}}(351+11504 q+\cdots), \\
& \tilde{f}_{2}^{2 A}(q)=\tilde{f}_{2}^{\prime 2 A}(q)=q^{\frac{11}{30}}(79+2808 q+\cdots), \\
& \tilde{f}_{3}^{2 A}(q)=\tilde{f}_{3}^{\prime 2 A}(q)=q^{\frac{29}{30}}(1352+27729 q+\cdots) .
\end{aligned}
$$

Combined with the characters of three-state Potts model, it turns out that the twined characters (3.13) merged into the Mckay-Thompson series of class 2A.

$$
\begin{aligned}
j^{2 A}(\tau)= & f_{0}(\tau) \cdot \tilde{f}_{0}^{2 A}(\tau)+f_{1}(\tau) \cdot \tilde{f}_{1}^{2 A}(\tau)+f_{2}(\tau) \cdot \tilde{f}_{2}^{2 A}(\tau)+{f^{\prime}}_{2}(\tau) \cdot \tilde{f}_{2}^{\prime 2 A}(\tau) \\
& +f_{3}(\tau) \cdot \tilde{f}_{3}^{2 A}(\tau)+{f^{\prime}}^{\prime}{ }_{3}(\tau) \cdot \tilde{f}_{3}^{\prime 2 A}(\tau)
\end{aligned}
$$

As another example, twined characters for $g=3 E$ read

$$
\begin{aligned}
\tilde{f}_{0}^{3 E}(q) & =q^{-\frac{29}{30}}\left(1+616 q^{2}+7833 q^{3}+\cdots\right), \\
\tilde{f}_{1}^{3 E}(q) & =q^{\frac{19}{30}}(-77-1925 q+\cdots), \\
\tilde{f}_{2}^{3 E}(q) & =q^{\frac{11}{30}}(54 \alpha+1485 \alpha q+\cdots), \\
\tilde{f}_{2}^{\prime 3 E}(q) & =q^{\frac{11}{30}}(54 \bar{\alpha}+1485 \bar{\alpha} q+\cdots), \\
\tilde{f}_{3}^{3 E}(q) & =q^{\frac{29}{30}}(-297 \alpha-4158 \alpha q+\cdots), \\
\tilde{f}_{3}^{\prime 3 E}(q) & =q^{\frac{29}{30}}(-297 \bar{\alpha}-4158 \bar{\alpha} q+\cdots),
\end{aligned}
$$

where $\alpha=\frac{-1+i \sqrt{3}}{2}$ and $\bar{\alpha}$ is its complex conjugate. Then, we get a new type of bilinear relation of the form,

$$
\begin{aligned}
j^{3 A}(\tau)= & f_{0}(\tau) \cdot \tilde{f}_{0}^{3 E}(\tau)+f_{1}(\tau) \cdot \tilde{f}_{1}^{3 E}(\tau)+f_{2}(\tau) \cdot \tilde{f}_{2}^{3 E}(\tau)+f_{2}^{\prime}(\tau) \cdot \tilde{f}_{2}^{\prime 3 E}(\tau) \\
& +f_{3}(\tau) \cdot \tilde{f}_{3}^{3 E}(\tau)+f_{3}^{\prime}(\tau) \cdot \tilde{f}_{3}^{\prime 3 E}(\tau)
\end{aligned}
$$


where $j^{3 A}(\tau)$ is Mckay-Thompson series of class $3 A$ given by,

$$
\begin{aligned}
j^{2 A}(\tau) & =\left(\left(\frac{\eta(q)}{\eta\left(q^{3}\right)}\right)^{12}+27\right)^{2} /\left(\frac{\eta(q)}{\eta\left(q^{3}\right)}\right)^{12}-42 \\
& =\frac{1}{q}+783 q+8672 q^{2}+65467 q^{3}+371520 q^{4}+\cdots .
\end{aligned}
$$

In appendix $\mathrm{C}$, we listed generalized bilinear relations that various twined characters satisfy. These suggest that the six-character rational CFT with $c=\frac{116}{5}$ dual to the critical three-state Potts model has $3 \cdot \mathrm{Fi}_{24}^{\prime}$ as an automorphism group.

\section{Dual of the critical Ising ${ }^{2}$ and $2 \cdot 2^{1+22} \cdot \mathrm{Co}_{2}$}

We now in turn consider a rational CFT dual to the tensor product of the critical Ising model, the simplest example of the $c=1$ CFTs studied in [22-24]. This theory has nine primaries of conformal weights

$$
h_{1}=h_{1}^{\prime}=\frac{1}{2}, h_{2}=h_{2}^{\prime}=\frac{1}{16}, h_{3}=1, h_{4}=h_{4}^{\prime}=\frac{9}{16}, h_{5}=\frac{1}{8},
$$

and their characters can be expressed by characters of the critical Ising model (2.2).

$$
\begin{aligned}
& g_{0}(\tau)=f_{0}(\tau) \cdot f_{0}(\tau), \quad g_{1}(\tau)=g_{1}^{\prime}(\tau)=f_{0}(\tau) \cdot f_{\epsilon}(\tau), \\
& g_{2}(\tau)=g_{2}^{\prime}(\tau)=f_{0}(\tau) \cdot f_{\sigma}(\tau), \quad g_{3}(\tau)=f_{\epsilon}(\tau) \cdot f_{\epsilon}(\tau), \\
& g_{4}(\tau)=g_{4}^{\prime}(\tau)=f_{\sigma}(\tau) \cdot f_{\epsilon}(\tau), \quad g_{5}(\tau)=f_{\sigma}(\tau) \cdot f_{\sigma}(\tau)
\end{aligned}
$$

The $9 \times 9$ extended modular matrix $\mathbb{S}$ reads,

$$
\mathbb{S}=\frac{1}{4}\left(\begin{array}{ccccccccc}
1 & 1 & 1 & \sqrt{2} & \sqrt{2} & 1 & \sqrt{2} & \sqrt{2} & 2 \\
1 & 1 & 1 & \sqrt{2} & -\sqrt{2} & 1 & \sqrt{2} & -\sqrt{2} & -2 \\
1 & 1 & 1 & -\sqrt{2} & \sqrt{2} & 1 & -\sqrt{2} & \sqrt{2} & -2 \\
\sqrt{2} & \sqrt{2} & -\sqrt{2} & 2 & 0 & -\sqrt{2} & -2 & 0 & 0 \\
\sqrt{2} & -\sqrt{2} & \sqrt{2} & 0 & 2 & -\sqrt{2} & 0 & -2 & 0 \\
1 & 1 & 1 & -\sqrt{2} & -\sqrt{2} & 1 & -\sqrt{2} & -\sqrt{2} & 2 \\
\sqrt{2} & \sqrt{2} & -\sqrt{2} & -2 & 0 & -\sqrt{2} & 2 & 0 & 0 \\
\sqrt{2} & -\sqrt{2} & \sqrt{2} & 0 & -2 & -\sqrt{2} & 0 & 2 & 0 \\
2 & -2 & -2 & 0 & 0 & 2 & 0 & 0 & 0
\end{array}\right),
$$

thus the product of two critical Ising model admit diagonal modular invariants of the form

$$
\begin{aligned}
Z(\tau, \bar{\tau})= & \left|g_{0}(\tau)\right|^{2}+\left|g_{1}(\tau)\right|^{2}+\left|g_{1}^{\prime}(\tau)\right|^{2}+\left|g_{2}(\tau)\right|^{2}+\left|g_{2}^{\prime}(\tau)\right|^{2} \\
& +\left|g_{3}(\tau)\right|^{2}+\left|g_{4}(\tau)\right|^{2}+\left|g_{4}^{\prime}(\tau)\right|^{2}+\left|g_{5}(\tau)\right|^{2} .
\end{aligned}
$$

We assumed that the characters (4.2) satisfy a bilinear relation

$$
\begin{aligned}
j(\tau)-744= & g_{0}(\tau) \tilde{g}_{0}(\tau)+g_{1}(\tau) \tilde{g}_{1}(\tau)+g_{1}^{\prime}(\tau) \tilde{g}_{1}^{\prime}(\tau)+g_{2}(\tau) \tilde{g}_{2}(\tau)+g_{2}^{\prime}(\tau) \tilde{g}_{2}^{\prime}(\tau) \\
& +g_{3}(\tau) \tilde{g}_{3}(\tau)+g_{4}(\tau) \tilde{g}_{4}(\tau)+g_{4}^{\prime}(\tau) \tilde{g}_{4}^{\prime}(\tau)+g_{5}(\tau) \tilde{g}_{5}(\tau)
\end{aligned}
$$


where the characters of a dual CFT with weights

$$
\tilde{h}_{1}=\tilde{h}_{1}^{\prime}=\frac{3}{2}, \tilde{h}_{2}=\tilde{h}_{2}^{\prime}=\frac{31}{16}, \tilde{h}_{3}=1, \tilde{h}_{4}=\tilde{h}_{4}^{\prime}=\frac{23}{16}, \tilde{h}_{5}=\frac{15}{8} .
$$

are the solutions of a differential equation,

$$
\begin{aligned}
0=[ & \mathcal{D}_{\tau}^{6}+\mu_{1} E_{4}(\tau) \mathcal{D}_{\tau}^{4}+\mu_{2} E_{6}(\tau) \mathcal{D}_{\tau}^{3}+\mu_{3} E_{4}^{2}(\tau) \mathcal{D}_{\tau}^{2}+\mu_{4} E_{4}(\tau) E_{6}(\tau) \mathcal{D}_{\tau} \\
& \left.+\mu_{5} E_{4}^{3}(\tau)+\mu_{6} E_{6}^{2}(\tau)+\mu_{7} \frac{E_{4}^{2}(\tau)}{E_{6}(\tau)} \mathcal{D}_{\tau}^{5}+\mu_{8} \frac{E_{4}^{3}(\tau)}{E_{6}(\tau)} \mathcal{D}_{\tau}^{3}+\mu_{9} \frac{E_{4}^{4}(\tau)}{E_{6}(\tau)} \mathcal{D}_{\tau}\right] \tilde{g}(\tau)
\end{aligned}
$$

However, inserting $q$-expansion of the characters into (4.7) does not determine all $\mu_{i}$ in (4.7), because it give us six constraints while there are nine unfixed parameters in (4.7). To remedy it, we compared two bilinear relations (4.5) and (2.4), which eventually provide us three additional constraints

$$
\begin{aligned}
& \tilde{f}_{0}(\tau)=f_{0}(\tau) \tilde{g}_{0}(\tau)+f_{\epsilon}(\tau) \tilde{g}_{1}(\tau)+f_{\sigma}(\tau) \tilde{g}_{2}(\tau) \\
& \tilde{f}_{\epsilon}(\tau)=f_{0}(\tau) \tilde{g}_{1}^{\prime}(\tau)+f_{\epsilon}(\tau) \tilde{g}_{3}(\tau)+f_{\sigma}(\tau) \tilde{g}_{4}(\tau) \\
& \tilde{f}_{\sigma}(\tau)=f_{0}(\tau) \tilde{g}_{2}^{\prime}(\tau)+f_{\sigma}(\tau) \tilde{g}_{4}^{\prime}(\tau)+f_{\epsilon}(\tau) \tilde{g}_{5}(\tau)
\end{aligned}
$$

Now one can fix nine parameters $\mu_{i}$ combining three equations (4.8) with six constraints from (4.7). In this way, we find that the nine parameters $\mu_{i}$ read,

$$
\begin{aligned}
& \mu_{1}=\frac{2647 \pi^{2}}{576}, \quad \mu_{2}=-i \frac{3495263687743883 \pi^{3}}{140037228850176}, \quad \mu_{3}=-\frac{598979 \pi^{4}}{82944}, \\
& \mu_{4}=-i \frac{779163580240684865 \pi^{5}}{20165360954425344}, \quad \mu_{5}=\frac{634818358457751325 \pi^{6}}{13443573969616896}, \\
& \mu_{6}=-\frac{5641332583789180993 \pi^{6}}{120992165726552064}, \quad \mu_{7}=i \pi, \\
& \mu_{8}=i \frac{810490346954549 \pi^{3}}{46679076283392}, \quad \mu_{9}=i \frac{284686915225948007 \pi^{5}}{6721786984808448} .
\end{aligned}
$$

As a result, we finally get below six different characters of dual CFT.

$$
\begin{aligned}
& \tilde{g}_{0}(\tau)=q^{-\frac{23}{24}}\left(1+46851 q^{2}+4310154 q^{3}+155027130 q^{4}+\cdots\right) \\
& \tilde{g}_{1}(\tau)=\tilde{g}_{1}^{\prime}(\tau)=q^{\frac{13}{24}}\left(2300+529828 q+28051444 q^{2}+\cdots\right) \\
& \tilde{g}_{2}(\tau)=\tilde{g}_{2}^{\prime}(\tau)=q^{\frac{47}{48}}\left(47104+4757504 q+178382848 q^{2}+\cdots\right) \\
& \tilde{g}_{3}(\tau)=q^{\frac{1}{24}}\left(23+46598 q+4311948 q^{2}+155017746 q^{3}+\cdots\right) \\
& \tilde{g}_{4}(\tau)=\tilde{g}_{4}^{\prime}(\tau)=q^{\frac{23}{48}}\left(2048+565248 q+31700992 q^{2}+\cdots\right) \\
& \tilde{g}_{5}(\tau)=q^{\frac{11}{12}}\left(47104+5230592 q+204855296 q^{2}+4630417408 q^{3}+\cdots\right)
\end{aligned}
$$

One can easily show that every coefficient in (4.10) can be expressed as a sum of the dimension of the irreducible representation of $2 \cdot 2^{1+22} \cdot \mathrm{Co}_{2} \cdot 2 \cdot 2^{1+22} \cdot \mathrm{Co}_{2}$ is a maximal subgroup of the baby Monster group. Its order is $2^{15} \cdot 3^{6} \cdot 5^{3} \cdot 7 \cdot 11 \cdot 23$. This multi-covering of the second largest Conway group $\mathrm{Co}_{2}$ has irreducible representations that includes $\mathbf{2 3}$, 
2048, 2300 and 47104. These numbers are in perfect agreement with the first terms of $\tilde{g}_{3}(\tau), \tilde{g}_{4}(\tau), \tilde{g}_{1}(\tau), \tilde{g}_{2}(\tau)$ and $\tilde{g}_{5}(\tau)$. Referring the table 1 , the other numbers in the characters can be decomposed as follow,

$$
\begin{aligned}
46851 & =\mathbf{1} \oplus \mathbf{2 7 5} \oplus \mathbf{4 6 5 7 5}, \quad 529828=\mathbf{2 3 0 0} \oplus \mathbf{5 0 6 0 0} \oplus \mathbf{4 7 6 9 2 8}, \\
4757504 & =2 \cdot \mathbf{4 7 1 0 4} \oplus \mathbf{4 6 6 3 2 9 6}, \quad 46598=\mathbf{2 3} \oplus \mathbf{4 6 5 7 5}, \\
565248 & =\mathbf{2 0 4 8} \oplus \mathbf{5 6 3 2 0 0}, \\
5230592 & =\mathbf{2 0 4 8} \oplus \mathbf{4 7 1 0 4} \oplus \mathbf{5 1 8 1 4 4} \oplus \mathbf{4 6 6 3 2 9 6} \\
4310154 & =\mathbf{1} \oplus \mathbf{2 5 3} \oplus \mathbf{2 7 5} \oplus \mathbf{4 6 5 7 5} \oplus \mathbf{1 0 2 4 6 5 0} \oplus \mathbf{3 2 3 8 4 0 0},
\end{aligned}
$$

where the first term in each decomposition can be again understood as the Virasoro descendent of the corresponding primary state. Thus we conjecture that the nine-character rational CFT of $c=23$, dual to the product of two critical Ising model, has $2 \cdot 2^{1+22} \cdot \mathrm{Co}_{2}$ as an automorphism group.

The modular S-matrix of the $c=23$ CFT is identical to the (4.3), because of the bilinear relation (4.5). Therefore, the modular invariant partition function of $c=23 \mathrm{CFT}$ is given by

$$
\begin{aligned}
Z(\tau, \bar{\tau})= & \left|\tilde{g}_{0}(\tau)\right|^{2}+\left|\tilde{g}_{1}(\tau)\right|^{2}+\left|\tilde{g}_{1}^{\prime}(\tau)\right|^{2}+\left|\tilde{g}_{2}(\tau)\right|^{2}+\left|\tilde{g}_{2}^{\prime}(\tau)\right|^{2} \\
& +\left|\tilde{g}_{3}(\tau)\right|^{2}+\left|\tilde{g}_{4}(\tau)\right|^{2}+\left|\tilde{g}_{4}^{\prime}(\tau)\right|^{2}+\left|\tilde{g}_{5}(\tau)\right|^{2} .
\end{aligned}
$$

Also, the modular S-matrix (4.3) guarantees the positive integer fusion rule algebra coefficients.

We also propose that the characters of the above RCFT (4.10) obey intriguing bilinear relations with those of the critical Ising model (2.2) to give the baby Monster modules (2.7),

$$
\begin{aligned}
& \tilde{f}_{0}(\tau)=f_{0}(\tau) \tilde{g}_{0}(\tau)+f_{\epsilon}(\tau) \tilde{g}_{1}(\tau)+f_{\sigma}(\tau) \tilde{g}_{2}(\tau) \\
& \tilde{f}_{\epsilon}(\tau)=f_{0}(\tau) \tilde{g}_{1}(\tau)+f_{\epsilon}(\tau) \tilde{g}_{3}(\tau)+f_{\sigma}(\tau) \tilde{g}_{4}(\tau) \\
& \tilde{f}_{\sigma}(\tau)=f_{0}(\tau) \tilde{g}_{2}(\tau)+f_{\epsilon}(\tau) \tilde{g}_{4}(\tau)+f_{\sigma}(\tau) \tilde{g}_{5}(\tau)
\end{aligned}
$$

\section{Self-dual RCFT and $2 \cdot \mathrm{Co}_{1}$}

We discuss in this section a "self-dual" RCFT with $c=12$ whose torus partition function admits a natural decomposition in terms of dimensions of representation of the Conway group $2 \cdot \mathrm{Co}_{1}$.

The RCFT of our interest is self-dual in a sense that its three characters, denoted by $f_{0}(\tau), f_{1}(\tau)$ and $f_{2}(\tau)$, satisfy a bilinear relation giving, ${ }^{1}$

$$
j(\tau)+96=f_{0}^{2}(\tau)+f_{1}^{2}(\tau)+\frac{1}{2} f_{2}^{2}(\tau)
$$

\footnotetext{
${ }^{1}$ Strictly speaking, a bilinear relation (5.1) cannot be an example of Monster anatomy, because $j(\tau)+96$ is not the Monster module. Nonetheless, in this section, we discuss a self-dual RCFT of $c=12$ because partition function of this theory also exhibit moonshine for Conway group.
} 
with $\lambda(\tau)=\frac{\theta_{2}^{4}(\tau)}{\theta_{3}^{4}(\tau)}$ and

$$
\begin{aligned}
f_{0}(\tau) & =\left(-\frac{1}{2} \lambda^{3}(\tau)+\frac{3}{2} \lambda^{2}(\tau)-\frac{3}{2} \lambda(\tau)+2\right)\left(\frac{16}{\lambda(\tau)(1-\lambda(\tau))}\right) \\
& =q^{-1 / 2}\left(1+276 q+11202 q^{2}+184024 q^{3}+\cdots\right), \\
f_{1}(\tau) & =\frac{8 \lambda^{3}(\tau)}{\lambda(\tau)(1-\lambda(\tau))}, \\
& =2048 q\left(1+24 q+300 q^{2}+2624 q^{3}+\cdots\right), \\
f_{2}(\tau) & =\frac{16 \lambda^{3}(\tau)}{\lambda(\tau)(1-\lambda(\tau))}+24, \\
& =24+4096 q+98304 q^{2}+1228800 q^{3}+\cdots .
\end{aligned}
$$

Here $f_{0}(\tau)$ is the vacuum character while $f_{1}(\tau)$ and $f_{2}(\tau)$ are characters for primary states of conformal weight $h=\frac{3}{2}$ and $h=\frac{1}{2}$. These characters are three independent solutions to a modular differential equation below,

$$
\left[\partial_{\tau}^{3}-\frac{1}{2} E_{2}(\tau) \partial_{\tau}^{2}+\frac{1}{24}\left(E_{2}^{2}(\tau)-13 E_{4}(\tau)\right) \partial_{\tau}\right] f(\tau)=0
$$

For later convenience, let us define $f_{--}(\tau), f_{-+}(\tau)$ and $f_{+-}(\tau)$ as follows

$$
\begin{aligned}
f_{--}(\tau) & =f_{0}(\tau)+f_{1}(\tau), \\
& =q^{-1 / 2}\left(1+276 q+2048 q^{3 / 2}+11202 q^{2}+49152 q^{5 / 2}+\cdots\right), \\
f_{-+}(\tau) & =f_{0}(\tau)-f_{1}(\tau), \\
& =q^{-1 / 2}\left(1+276 q-2048 q^{3 / 2}+11202 q^{2}-49152 q^{5 / 2}+\cdots\right), \\
f_{+-}(\tau) & =f_{2}(\tau) \\
& =24+4096 q+98304 q^{2}+1228800 q^{3}+10747904 q^{4}+\cdots .
\end{aligned}
$$

From the modular S-matrix of the self-dual theory,

$$
\left(\begin{array}{l}
f_{--}(1-\lambda) \\
f_{-+}(1-\lambda) \\
f_{+-}(1-\lambda)
\end{array}\right)=\left(\begin{array}{lll}
1 & 0 & 0 \\
0 & 0 & 1 \\
0 & 1 & 0
\end{array}\right)\left(\begin{array}{l}
f_{--}(\lambda) \\
f_{-+}(\lambda) \\
f_{+-}(\lambda)
\end{array}\right)
$$

one can show that the $\mathrm{SL}(2, \mathbb{Z})$ invariant partition function would be

$$
Z=\frac{1}{2}\left(\left|f_{--}(\tau)\right|^{2}+\left|f_{-+}(\tau)\right|^{2}+\left|f_{+-}(\tau)\right|^{2}\right)+\text { const. }
$$

Notice here that the character $f_{--}(\tau)$ is nothing but the Neveu-Schwarz (NS) partition function $K(\tau)$ of $\mathcal{N}=1$ extremal superconformal theory [17]. In fact, one can understand from (5.6) that the RCFT with $c=12$ of our interest is the GSO projection of the $\mathcal{N}=1$ 
extremal SCFT where

$$
\begin{aligned}
& f_{--}(\tau)=\operatorname{tr}_{\mathrm{NS}}\left[q^{L_{0}-c / 24}\right], \\
& f_{-+}(\tau)=\operatorname{tr}_{\mathrm{NS}}\left[(-1)^{F} q^{L_{0}-c / 24}\right], \\
& f_{+-}(\tau)=\operatorname{tr}_{\mathrm{R}}\left[q^{L_{0}-c / 24}\right]
\end{aligned}
$$

and the constant term corresponding to the Witten index, $\operatorname{tr}_{\mathrm{R}}\left[(-1)^{F} q^{L_{0}-c / 24} \bar{q}^{\bar{L}_{0}-c / 24}\right]=$ $24^{2}$. Here the each trace can be performed in either the NS Hilbert space or the Ramond Hilbert space of the $\mathcal{N}=1$ SCFT. This SCFT was first made by Frenkel, Lepowsky and Meurman [2], and revisited later by Duncan [3, 8].

The $\mathcal{N}=1$ extremal SCFT with $c=12$ is well-known to have $2 \cdot \mathrm{Co}_{1}=\mathrm{Co}_{0}$ as an automorphism group. After the GSO projection, the double covering of the largest Conway group continues to serve as an automorphism group of the self-dual theory. The Virasoro character decomposition of the partition further gives new evidence that the automorphism group of the RCFT with $c=12$ may be enhanced to a larger group $2^{1+24} \cdot \mathrm{Co}_{1}$, a maximal subgroup of the Monster group. To see this, let us decompose the partition function (5.6) in terms of the $c=12$ Virasoro characters,

$$
\begin{aligned}
Z(\tau, \bar{\tau})= & \chi_{0}(q) \bar{\chi}_{0}(q)+276\left(\chi_{0}(\bar{q}) \bar{\chi}_{1}(\bar{q})+\text { c.c. }\right)+76176 \chi_{1}(\bar{q}) \bar{\chi}_{1}(\bar{q}) \\
& +10925\left(\chi_{0}(\bar{q}) \bar{\chi}_{2}(\bar{q})+\text { c.c. }\right)+3015300\left(\chi_{1}(\bar{q}) \bar{\chi}_{2}(\bar{q})+\text { c.c. }\right) \\
& +1081344\left(\chi_{\frac{1}{2}}(q) \bar{\chi}_{\frac{5}{2}}(\bar{q})+\text { c.c. }\right)+49152 \chi_{\frac{1}{2}}(\bar{q}) \bar{\chi}_{\frac{1}{2}}(\bar{q})+\cdots
\end{aligned}
$$

It turns out that every coefficients in (5.8) is related to the irreducible representations of $2^{1+24} \cdot \mathrm{Co}_{1}$. Some details of the number decomposition are presented below.

$$
\begin{aligned}
49152 & =\mathbf{1} \oplus 2 \cdot \mathbf{2 7 6} \oplus \mathbf{2 9 9} \oplus \mathbf{1 7 7 1} \oplus \mathbf{8 8 5 5} \oplus \mathbf{3 7 6 7 4}, \\
276 & =\mathbf{2 3} \oplus \mathbf{2 5 3}, \quad 10925 \mathbf{=} \mathbf{2 9 9} \oplus \mathbf{1 7 7 1} \oplus \mathbf{8 8 5 5} \oplus \mathbf{1 0 6 2 6}, \\
76176 & =\mathbf{1} \oplus \mathbf{2 7 6} \oplus \mathbf{2 9 9} \oplus \mathbf{1 7 7 1} \oplus \mathbf{8 8 5 5} \oplus \mathbf{2 7 3 0 0} \oplus \mathbf{3 7 6 7 4}
\end{aligned}
$$

\section{Discussion}

It is known that the CFT of $c=1$ discussed in section 4 allows a non-diagonal partition function

$$
Z(\tau, \bar{\tau})=\left|g_{0}(\tau)+g_{3}(\tau)\right|^{2}+\left|g_{1}(\tau)+g_{1}^{\prime}(\tau)\right|^{2}+2\left|g_{5}(\tau)\right|^{2},
$$

different from (4.4). (6.1) is the partition function of another example of $c=1$ CFT studied in $[23,24]$. One can show that the characters in (6.1) can obey a new bilinear relation

$$
\begin{aligned}
j(\tau)-720= & \left(g_{0}(\tau)+g_{3}(\tau)\right)\left(\tilde{g}_{0}(\tau)+\tilde{g}_{3}(\tau)\right)+\left(g_{1}(\tau)+g_{1}^{\prime}(\tau)\right)\left(\tilde{g}_{1}(\tau)+\tilde{g}_{1}^{\prime}(\tau)\right) \\
& +2 g_{5}(\tau) \tilde{g}_{5}(\tau),
\end{aligned}
$$


from which one can read the partition function of the dual theory

$$
\tilde{Z}(\tau, \bar{\tau})=\left|\tilde{g}_{0}(\tau)+\tilde{g}_{3}(\tau)\right|^{2}+\left|\tilde{g}_{1}(\tau)+\tilde{g}_{1}^{\prime}(\tau)\right|^{2}+2\left|\tilde{g}_{5}(\tau)\right|^{2}
$$

Notice that (6.3) can be understood as a non-diagonal partition function of the rational CFT of $c=23$ discussed in section 4. It is obvious that the dual CFT also exhibit the moonshine for $2 \cdot 2^{1+22} \cdot \mathrm{Co}_{2}$. This is somewhat trivial, because it shares the characters $(4.10)$ as building blocks. However, above example cannot be considered as a class of the Monster anatomy, because $j(\tau)-720$ is not the Monster module.

It has been proposed that the Hecke images of the vector-valued modular form can construct a set of admissible characters for specific RCFT [25]. The Hecke image $T_{p} f_{i}(\tau)$ is characterized by the conductor $N$ and a natural number $p$ that is relatively prime to $N$. As an example, it has been known that the conductor of the critical Ising model is given by $N=48$. Then, one can show that the Hecke images for $p=47$ are exactly agree to the characters of the baby Monster CFT, namely (2.7). In similar way, it is easy to see that the conductor of three-state Potts model and the tensor product of the critical Ising model are given by $N=30$ and $N=24$, respectively. We checked that the characters (3.10) can be considered as the Hecke images of (3.4) with $p=29$. In similar way, we found that the vector-valued modular form $\left(\tilde{g}_{0}(\tau)+\tilde{g}_{3}(\tau), \tilde{g}_{1}(\tau)+\tilde{g}_{1}^{\prime}(\tau), \tilde{g}_{5}(\tau)\right)$ in $(6.3)$ is also realized as the Hecke images of $\left(g_{0}(\tau)+g_{3}(\tau), g_{1}(\tau)+g_{1}^{\prime}(\tau), g_{5}(\tau)\right)$ with $p=23$. However, it turns out that the Hecke images of (4.2) cannot generate the six characters in (4.10). More precisely, the characters $\tilde{g_{2}}(\tau)$ and $\tilde{g_{4}}(\tau)$ in $(4.10)$ are not able to realized as the Hecke images of the characters in (4.2).

One can also ask if the dual CFT of the tricritical Ising model, the next simplest unitary minimal model $\mathcal{M}(5,4)$, can exhibit the moonshine phenomena. However, it is nontrivial to obtain the characters of the candidate dual CFT with $c=\frac{233}{10}$. This is partially because one cannot determine all free parameters in the corresponding MDE completely from the known CFT data [12]. We tried to find the dual characters of $c=\frac{233}{10}$ CFT using the Hecke operator of $N=240$ and $p=233$, however it turns out that this Hecke operator does not produce admissible characters.

On the other hand, it is known that the tricritical Ising model is endowed with the $\mathcal{N}=1$ supersymmetry. The NS partition function of the model can be contributed by the NS superconformal characters of the vacuum and the primary state of $h=\frac{1}{10}$. It would be interesting to search for a rational SCFT whose NS characters obey a new bilinear relation with those of the tricritical Ising model to give the $K(\tau)$-function [17]. This new bilinear relation would lead to a picture of the Conway group decomposition, instead of the Monster group decomposition. We leave them as a future project.

The bigger challenge is to find all the two-dimensional dual CFT pairs for the rest of the sporadic groups and understand the origin of bilinear relations. In particular, it would be extremely interesting to see if a CFT dual to '(three-state Potts model $) \times($ a certain CFT)' can show moonshine for the multiple-covering of the largest Mathieu group, $2^{12} \cdot \mathrm{M}_{24}$. This is analogous to the idea used to find the dual CFT with $2 \cdot 2^{1+22} \cdot \mathrm{Co}_{2}$ and correspond to the green arrow in figure 1. 


\section{Acknowledgments}

We would like to thank Hyun Kyu Kim for useful discussions. We are grateful to the anonymous referee of JHEP for giving us numerous, invaluable comments on the manuscript. KL is supported in part by the National Research Foundation of Korea Grant NRF2017R1D1A1B06034369. KL would like to thank the colleagues at IIP Natal Brazil where the part of work is done. The research of S.L. is supported in part by the National Research Foundation of Korea (NRF) Grant NRF-2017R1C1B1011440. K.L. and S.L. thank the Aspen Center for Physics (supported by National Science Foundation grant PHY-1607611).

\section{A Dimension of the irreducible representations}

Here we give a partial list of the dimension of the irreducible representations for various sporadic groups.

\begin{tabular}{|c|c|}
\hline & Dimension of the irreducible representations \\
\hline $2 \cdot \mathbb{B}$ & $\begin{array}{l}\{1,4371,96255,96256,1139374,9458750,9550635,10506240 \\
63532485,347643114,356054375,410132480,1407126890 \\
3214743741,4221380670,4275362520,4622913750, \cdots\}\end{array}$ \\
\hline $3 \cdot \mathrm{Fi}_{24}^{\prime}$ & $\begin{array}{l}\{1,783,8671,57477,64584,249458,306153,555611,1603525, \\
1666833,4864431,6724809,19034730,25356672,32715683, \\
35873145,40536925,43779879,48893768,74837400, \cdots,\}\end{array}$ \\
\hline $2 \cdot \mathrm{Co}_{1}$ & $\begin{array}{l}\{1,24,276,299,1771,2024,2576,4576,8855,17250,27300,37674, \\
40480,44275,80730,94875,95680,170016,299000,313950,315744, \\
345345,351624,376740,388080,483000,644644,673750,789360, \\
822250,871884,1434510,1450449,1771000,1821600,1841840, \cdots\}\end{array}$ \\
\hline $2^{1+24} \cdot \mathrm{Co}_{1}$ & $\begin{array}{l}\{1,276,299,1771,8855,17250,27300,37674,44275,80730,94875 \\
98280,98304,313950,345345,376740,483000,644644,673750 \\
822250,871884,1434510,1450449,1771000,1821600,2055625 \\
2260440,2417415,2464749,2464749,2816856,2877875, \cdots\}\end{array}$ \\
\hline $2^{1+22} \cdot \mathrm{Co}_{2}$ & $\begin{array}{l}\{1,23,253,275,1771,2024,2048,2277,2300,4025,7084,9625, \\
10395,12650,23000,31625,31878,37422,44275,46575,47104, \\
50600,63250,91125,113850,129536,177100,184437,212520, \\
221375,226688,239085,245916,253000,284625,312984,368874, \\
398475,430353,442750,462000,467775,476928,518144,531300, \\
558900,563200,579600, \cdots\}\end{array}$ \\
\hline
\end{tabular}

Table 1. Dimension of the irreducible representation in various sporadic groups. The list of the representation read from GAP package [26]. 


\section{B Character tables of $2 \cdot \mathbb{B}$ and $3 \cdot \mathrm{Fi}_{24}^{\prime}$}

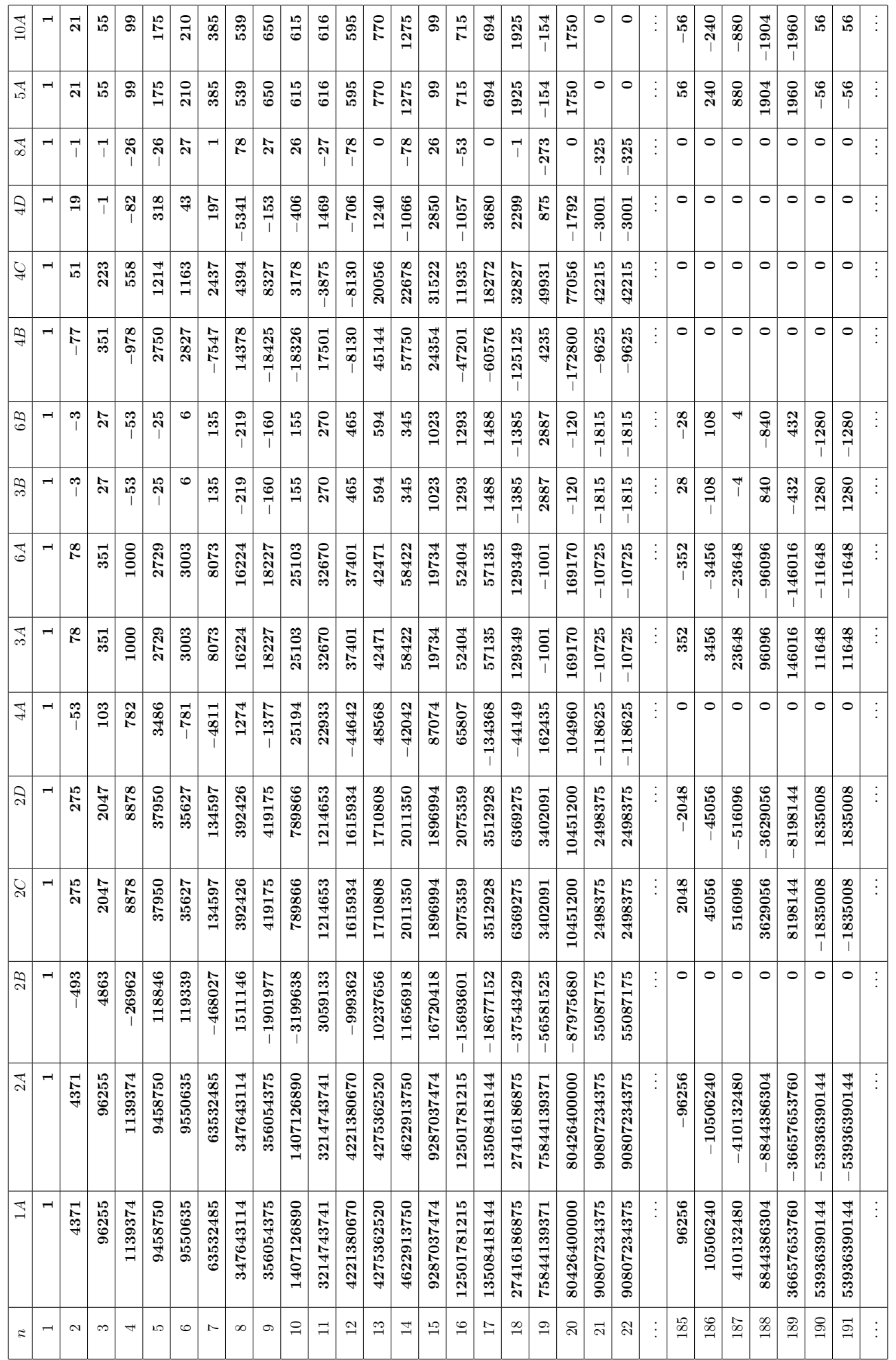

Table 2. Partial character table of the baby Monster group $2 \cdot \mathbb{B}$. 


\begin{tabular}{|c|c|c|c|c|c|c|c|c|c|c|c|c|c|c|c|c|c|}
\hline & $7 a$ & $\bar{\infty} \dot{\theta}$ & 8 & 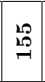 & 10 & $D$ & :े & & 兽 & & & & & : & & & 总 \\
\hline & -1 & $\vec{\infty}:$ & in & 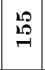 & 10 & 10 & & t. & คี & 兽 & สี & & & 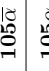 & & & 离 \\
\hline & $\Rightarrow:$ & $\vec{\infty} \dot{\theta}$ & 8 & $\mid \begin{array}{l}\text { 量 } \\
\stackrel{9}{9}\end{array}$ & 10 & $D$ & $\stackrel{ి}{2}$ & م2 & $\stackrel{12}{\rightarrow}$ & $\stackrel{20}{7}$ & $\mathfrak{\Sigma}$ & $\mathfrak{\Sigma}$ & & 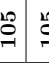 & & & 跑 \\
\hline & $\rightarrow$ & 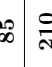 & $\stackrel{t}{\circ}$ & $\mid \begin{array}{l}\infty \\
10 \\
10\end{array}$ & $\frac{8}{1}$ & $\begin{array}{l}8 \\
1 \\
1\end{array}$ & \begin{tabular}{c|c}
$\hat{\infty}$ \\
$\infty$ \\
$\infty$
\end{tabular} & 6 & 논 & กั & | & స్ّ & 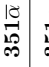 & $\begin{array}{l}8 \\
0\end{array}$ & 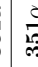 & & 总 \\
\hline 坣 & $\Rightarrow L$ & $\frac{1}{\infty}$ & 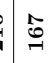 & $\mid \begin{array}{l}\infty \\
10 \\
10\end{array}$ & $\underset{7}{\stackrel{q}{7}}$ & $\frac{9}{7}$ & $\begin{aligned} & \hat{\infty} 1 \\
& \infty\end{aligned}$ & 6 & ลิ & $\underset{\text { N }}{\text { S }}$ & | & $\mid$ & 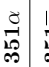 & 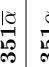 & 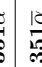 & & 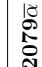 \\
\hline 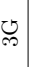 & $\Rightarrow L$ & $\frac{1}{\infty}$ & 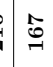 & $\mid \begin{array}{l}\infty \\
10 \\
10\end{array}$ & $\frac{q}{7}$ & $\frac{9}{7}$ & \begin{tabular}{r|r}
$\hat{\infty}$ \\
$\infty$
\end{tabular} & 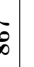 & ลे & ล & $\stackrel{0}{\sim}$ & & 路 & 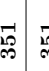 & 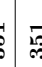 & & 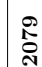 \\
\hline & $\rightarrow 1$ & $\hat{\imath}$ & $\mathscr{D}_{\infty}$ & 总 & 跑 & 苓 & $\begin{array}{l}\infty \\
\infty \\
0\end{array}$ & 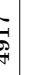 & 密 & 감 & 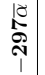 & 总 & 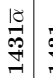 & 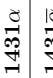 & 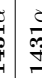 & 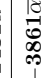 & 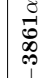 \\
\hline 팡 & $\Rightarrow N$ & $\begin{array}{r}\hat{T} \\
\mathbf{T}\end{array}$ & ${ }_{\infty}$ & 总 & 筮 & 苓 & $\begin{array}{c}\infty \\
0 \\
\infty \\
\infty\end{array}$ & 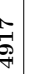 & 总 & $\mid$ & ר్⿱ & 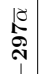 & 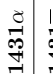 & 范 & $\frac{10}{\gamma}$ & 总 & 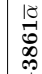 \\
\hline 全 & $\rightarrow$ & $\begin{array}{r}\hat{1} \\
>\end{array}$ & ${ }_{\infty}$ & 冬 & 答 & 跑 & 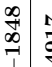 & 㕉 & $\overrightarrow{10}$ & If & 勇 & 勇 & $\overrightarrow{\mathfrak{g}}$ & 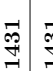 & $\bar{g}$ & $\mathbb{\infty}_{\infty}^{\infty}$ & \begin{tabular}{|l}
$\overrightarrow{0}$ \\
$\infty$ \\
$\infty$
\end{tabular} \\
\hline 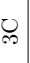 & $\rightarrow 7$ & 齐 & 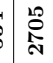 & 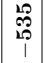 & 筤 & 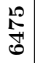 & 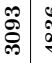 & 党 & 0 & 0 & 0 & 0 & 0. & 0 & 0 & 0 & 0 \\
\hline & $\rightarrow \approx$ & 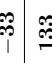 & R & 5 & $\frac{12}{p}$ & $\stackrel{2}{\infty}$ & 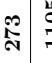 & 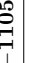 & 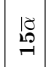 & 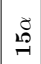 & ָָ & & 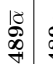 & 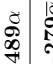 & 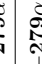 & 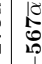 & 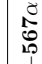 \\
\hline o & $\rightarrow \approx$ & 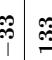 & $\stackrel{R}{R}$ & $\vec{\sigma}$ & $\frac{12}{0}$ & $\stackrel{2}{0}$ & 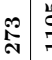 & 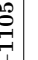 & 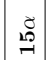 & 崷 & ָี & 존 & 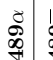 & 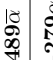 & 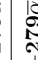 & 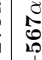 & $\begin{array}{l}1 \\
0 \\
0\end{array}$ \\
\hline 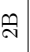 & $\Rightarrow \approx$ & 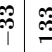 & $\mathbb{R}_{\infty}^{R}$ & 5 & 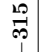 & 吕 & 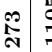 & 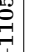 & 12 & 19 & ล & $\mathfrak{N}$ & 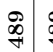 & 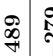 & 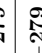 & 藏 & 管 \\
\hline$b$ & $\Rightarrow$ & $\begin{array}{ll}0 \\
0\end{array}$ & శ్ & $\mid \begin{array}{l}\infty \\
0 \\
0 \\
1\end{array}$ & 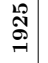 & 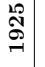 & $\begin{array}{l}\mathrm{b} \\
\mathrm{g}\end{array}$ & | & 莡 & 竜 & 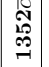 & 旋 & 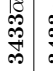 & 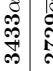 & 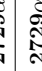 & 点 & 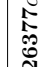 \\
\hline క్ర & $\rightarrow$ & 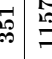 & 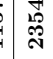 & $\mid$ & 旋 & 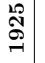 & 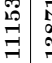 & 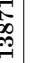 & 苟 & 落 & 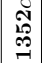 & 政 & 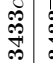 & 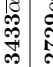 & & & \begin{tabular}{|l}
$\mathbb{R}$ \\
:
\end{tabular} \\
\hline త্స & - & 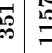 & $\sqrt{20}$ & 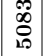 & 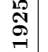 & జే & $\stackrel{i}{=}=$ & 露 & $\stackrel{R}{2}$ & i & $\mid$ & & 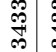 & 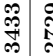 & & & 氺 \\
\hline & $\Rightarrow$ & $\begin{array}{ll}\mathbb{6} \\
0 \\
\infty \\
\infty\end{array}$ & & 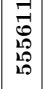 & 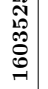 & 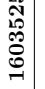 & 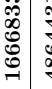 & |ר & 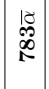 & 总 & $\mid$ & $\mid$ & 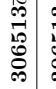 & 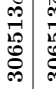 & & & \\
\hline & $\rightarrow \frac{5}{2}$ & & & 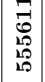 & 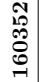 & 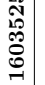 & 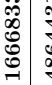 & 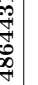 & 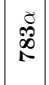 & $\begin{array}{l}\mathscr{L} \\
\substack{\infty \\
1 \\
1}\end{array}$ & 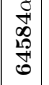 & & 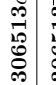 & $\stackrel{\mathscr{m}}{-1}$ & & & \\
\hline & - & 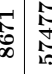 & L & 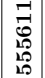 & 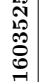 & 党 & $\begin{array}{l}0 \\
0 \\
: \\
: \\
:\end{array}$ & 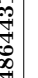 & 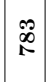 & 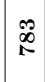 & $\mid$\begin{tabular}{|c}
$\overrightarrow{0}$ \\
0 \\
$d$ \\
0 \\
0
\end{tabular} & & $\begin{array}{l}0 \\
0 \\
0 \\
0 \\
0 \\
0\end{array}$ & 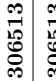 & & & \\
\hline & & & & & & s & & & & & $\exists$ & $\stackrel{ }{\exists}$ & $\stackrel{0}{\exists}$ & $\vec{\exists}$ & & & \\
\hline
\end{tabular}

Table 3. Partial character table of the Fischer group $3 \cdot \mathrm{Fi}_{24}^{\prime}$. Here $\alpha=\frac{-1+i \sqrt{3}}{2}$ and $\bar{\alpha}=\frac{-1-i \sqrt{3}}{2}$. 


\begin{tabular}{|c|c|c|c|c|c|}
\hline$g$ & $g_{\mathbb{M}}$ & $g$ & $g_{\mathbb{M}}$ & $g$ & $g_{\mathbb{M}}$ \\
\hline $2 \mathrm{~A}$ & $2 A$ & $3 \mathrm{~B}$ & $3 \mathrm{~B}$ & $6 \mathrm{~A}$ & $6 \mathrm{~A}$ \\
\hline $2 \mathrm{~B}$ & $2 A$ & $4 \mathrm{~A}$ & $4 \mathrm{~B}$ & $6 \mathrm{~B}$ & $6 \mathrm{D}$ \\
\hline $2 \mathrm{C}$ & $2 A$ & $4 \mathrm{~B}$ & $4 \mathrm{~A}$ & $8 \mathrm{~A}$ & $8 \mathrm{C}$ \\
\hline $2 \mathrm{D}$ & $2 B$ & $4 \mathrm{D}$ & $4 \mathrm{C}$ & $5 \mathrm{~A}$ & $5 \mathrm{~A}$ \\
\hline $3 \mathrm{~A}$ & $3 A$ & $4 \mathrm{C}$ & $4 \mathrm{~A}$ & $10 \mathrm{~A}$ & $10 \mathrm{~A}$ \\
\hline
\end{tabular}

Table 4. Generalized bilinear relation for $2 \cdot \mathbb{B}$.

\begin{tabular}{|c|c|c|c|c|c|}
\hline$g$ & $g_{\mathbb{M}}$ & $g$ & $g_{\mathbb{M}}$ & $g$ & $g_{\mathbb{M}}$ \\
\hline $3 \mathrm{~A}, 3 \mathrm{~B}$ & $3 A$ & $6 \mathrm{C}, 6 \mathrm{D}$ & $6 \mathrm{C}$ & $3 \mathrm{G}$ & $3 \mathrm{~A}$ \\
\hline $2 \mathrm{~A}$ & $2 A$ & $3 \mathrm{C}$ & $3 \mathrm{~A}$ & $3 \mathrm{H}, 3 \mathrm{I}$ & $3 \mathrm{~B}$ \\
\hline $6 \mathrm{~A}, 6 \mathrm{~B}$ & $6 A$ & $3 \mathrm{D}$ & $3 \mathrm{~B}$ & $4 \mathrm{~A}$ & $4 \mathrm{~A}$ \\
\hline $2 \mathrm{~B}$ & $2 B$ & $3 \mathrm{E}, 3 \mathrm{~F}$ & $3 \mathrm{~A}$ & $12 \mathrm{~A}, 12 \mathrm{~B}$ & $12 \mathrm{~A}$ \\
\hline
\end{tabular}

Table 5. Generalized bilinear relation for $3 \cdot \mathrm{Fi}_{24}^{\prime}$.

\section{Generalized bilinear relations}

\section{C.1 $2 \cdot \mathbb{B}$}

We find the twined characters of baby Monster CFT for various $g \in 2 \cdot \mathbb{B}$ which are combined with the characters of Ising models and form the Mckay-Thompson series for various $g_{\mathbb{M}} \in \mathbb{M}$. More preciesly, general expression of generalized bilinear relation have a form of

$$
j^{g_{\mathbb{M}}}(\tau)=f_{0}(\tau) \cdot \tilde{f}_{0}^{g}(\tau)+f_{\epsilon}(\tau) \cdot \tilde{f}_{\epsilon}^{g}(\tau)+f_{\sigma}(\tau) \cdot \tilde{f}_{\sigma}^{g}(\tau),
$$

where $\tilde{f}^{g}(\tau)$ is twined character for $g \in 2 \cdot \mathbb{B}$ and $j^{g_{\mathbb{M}}}(\tau)$ denotes Mckay-Thompson series for class $g_{\mathbb{M}} \in \mathbb{M}$. Table 4 present which twined character forming Mckay-Thompson series of type $g_{\mathbb{M}}$. For instance, once we have twined characters of baby Monster CFT for $g=2 C$, they merge with the characters of Ising model to produce Mckay-Thompson series of class $2 A$.

\section{$\mathrm{C.2} 3 \cdot \mathrm{Fi}_{24}^{\prime}$}

It turns out that the twined characters of $c=\frac{116}{5}$ putative CFT for various $g \in 3 \cdot \mathrm{Fi}_{24}^{\prime}$ also constitute the Mckay-Thompson series of certain class $g_{\mathbb{M}} \in \mathbb{M}$ with the characters of three-states Potts model. We find that the explicit form of the generalized bilinear relation is given by

$$
\begin{aligned}
j^{g \mathbb{M}}(\tau)= & f_{0}(\tau) \cdot \tilde{f}_{0}^{g}(\tau)+f_{1}(\tau) \cdot \tilde{f}_{1}^{g}(\tau)+f_{2}(\tau) \cdot \tilde{f}_{2}^{g}(\tau)+f_{2}^{\prime}(\tau) \cdot \tilde{f}_{2}^{\prime g}(\tau) \\
& +f_{3}(\tau) \cdot \tilde{f}_{3}^{g}(\tau)+f_{3}^{\prime}(\tau) \cdot \tilde{f}_{3}^{\prime g}(\tau)
\end{aligned}
$$

where $\tilde{f}^{g}(\tau)$ is twined character for $g \in 3 \cdot \mathrm{Fi}_{24}^{\prime}$ and $j^{g_{\mathbb{M}}}(\tau)$ denotes Mckay-Thompson series for class $g_{\mathbb{M}} \in \mathbb{M}$ as before. Table 5 exhibit which twined character yields Mckay-Thompson series of certain class, like table 4 describes. 
Open Access. This article is distributed under the terms of the Creative Commons Attribution License (CC-BY 4.0), which permits any use, distribution and reproduction in any medium, provided the original author(s) and source are credited.

\section{References}

[1] J.H. Conway and S.P. Norton, Monstrous Moonshine, Bull. London Math. Soc. 11 (1979) 308.

[2] I. Frenkel, J. Lepowsky and A. Meurman, Vertex operator algebras and the Monster, Academic Press, U.S.A. (1988).

[3] J.F. Duncan, Super-moonshine for conway's largest sporadic group, math/0502267.

[4] T. Eguchi, H. Ooguri and Y. Tachikawa, Notes on the K3 surface and the Mathieu group $M_{24}$, Exper. Math. 20 (2011) 91 [arXiv:1004.0956] [INSPIRE].

[5] M.C.N. Cheng, J.F.R. Duncan and J.A. Harvey, Umbral Moonshine and the Niemeier lattices, arXiv:1307.5793 [INSPIRE].

[6] M.C.N. Cheng, J.F.R. Duncan and J.A. Harvey, Umbral Moonshine, Commun. Num. Theor. Phys. 08 (2014) 101 [arXiv:1204.2779] [INSPIRE].

[7] M.C.N. Cheng et al., Mock modular Mathieu moonshine modules, arXiv:1406.5502 [INSPIRE].

[8] J.F.R. Duncan and S. Mack-Crane, The Moonshine module for Conway's Group, SIGMA 3 (2015) e10 [arXiv:1409.3829] [INSPIRE].

[9] M.C.N. Cheng, S.M. Harrison, S. Kachru and D. Whalen, Exceptional algebra and sporadic groups at $c=12$, arXiv: 1503.0721 .

[10] J.A. Harvey and B.C. Rayhaun, Traces of singular moduli and Moonshine for the Thompson group, Commun. Num. Theor. Phys. 10 (2016) 23 [arXiv:1504.0817].

[11] J.F.R. Duncan, M.H. Mertens and K. Ono, O'nan moonshine and arithmetic, arXiv: 1702.03516.

[12] H.R. Hampapura and S. Mukhi, Two-dimensional RCFTs without Kac-Moody symmetry, JHEP 07 (2016) 138 [arXiv: 1605.0331].

[13] J.B. Bae, S. Lee and J. Song, Modular constraints on conformal field theories with currents, JHEP 12 (2017) 045.

[14] A.R. Chandra and S. Mukhi, Towards a classification of two-character rational conformal field theories, arXiv:1810.0947.

[15] S.D. Mathur, S. Mukhi and A. Sen, On the classification of rational conformal field theories, Phys. Lett. B 213 (1988) 303 [INSPIRE].

[16] R.A. Wilson, Fischer's monsters, https://www.math.uni-bielefeld.de/ baumeist/wop2017/slides/Fischer80.pdf.

[17] E. Witten, Three-dimensional gravity revisited, arXiv:0706.3359 [INSPIRE].

[18] G. Hoehn, Selbstduale vertexoperatorsuperalgebren und das babymonster (self-dual vertex operator super algebras and the baby monster), arXiv:0706.0236. 
[19] M.R. Gaberdiel, S. Hohenegger and R. Volpato, Mathieu twining characters for K3, JHEP 09 (2010) 058 [arXiv: 1006.0221] [INSPIRE].

[20] T. Eguchi and K. Hikami, Note on twisted elliptic genus of K3 surface, Phys. Lett. B 694 (2011) 446 [arXiv: 1008.4924] [INSPIRE].

[21] P. Di Francesco, P. Mathieu and D. Senechal, Conformal field theory, Graduate texts in contemporary physics. Springer, Germany (1997).

[22] S. Elitzur, E. Gross, E. Rabinovici and N. Seiberg, Aspects of bosonization in string theory, Nucl. Phys. B 283 (1987) 413 [INSPIRE].

[23] P.H. Ginsparg, Curiosities at $c=1$, Nucl. Phys. B 295 (1988) 153 [InSPIRE].

[24] R. Dijkgraaf, C. Vafa, E.P. Verlinde and H.L. Verlinde, The operator algebra of orbifold models, Commun. Math. Phys. 123 (1989) 485 [INSPIRE].

[25] J.A. Harvey and Y. Wu, Hecke relations in rational conformal field theory, JHEP 09 (2018) 032 [arXiv: 1804.0686].

[26] The GAP group, GAP - Groups, Algorithms, and Programming. Version 4.8.7 (2017). 